\title{
Correction to: Nocardia terrae sp. nov., an actinomycete isolated from soil in Thailand
}

\author{
Pawina Kanchanasin ${ }^{1} \cdot$ Masahiro Yuki ${ }^{2} \cdot$ Takuji Kudo $^{2} \cdot$ Moriya Ohkuma $^{2} \cdot$ Wongsakorn Phongsopitanun ${ }^{1}$. \\ Somboon Tanasupawat ${ }^{1}$ (D)
}

Published online: 29 May 2021

๑) Springer-Verlag GmbH Germany, part of Springer Nature 2021

\section{Correction to: \\ Archives of Microbiology (2021) 203:1071-1077 \\ https://doi.org/10.1007/s00203-020-02107-3}

In the original article, there is an error in the description of Nocardia terrae. The correct sentence is "The type strain, ET3-3 T (=JCM 33776 T=TISTR 2837 T), was isolated from soil collected from Chachoengsao province, Thailand".

Publisher's Note Springer Nature remains neutral with regard to jurisdictional claims in published maps and institutional affiliations.

The original article can be found online at https://doi.org/10.1007/ s00203-020-02107-3.

Somboon Tanasupawat

Somboon.T@chula.ac.th

1 Department of Biochemistry and Microbiology, Faculty

of Pharmaceutical Sciences, Chulalongkorn University, 254

Phayathai Road, Pathumwan, Bangkok 10330, Thailand

2 Japan Collection of Microorganisms, RIKEN BioResource

Center, 3-1-1 Koyadai, Tsukuba, Ibaraki 305-0074, Japan 